\title{
Hydraulic physical model production with Computer Numerically Controlled (CNC) manufacturing techniques.
}

\author{
Pierre-Yves Henry ${ }^{*}, 1$, Jochen Aberle ${ }^{2,1}$, Christy Ushanth Navaratnam ${ }^{1}$, and Nils Ruther ${ }^{1}$ \\ ${ }^{1}$ Department of Civil and Environmental Engineering, Norwegian University of Science and \\ Technology (NTNU) - S.P. Andersens vei 5, 7491 Trondheim, Norway \\ ${ }^{2}$ Leichtweiß Insititute for Hydraulic Engineering and Water Resources, Technische Universität \\ Braunschweig - Braunschweig, Germany
}

\begin{abstract}
Physical models are a well-accepted tool in hydraulic engineering, allowing for the detailed characterisation of flow processes and the validation of structure designs with complex boundary conditions. The methods used to construct physical models typically produce a surface roughness which does not necessarily scale with the surface roughness of the prototype. In this context, this paper discusses novel construction methods allowing a detailed reproduction of roughness elements in scaled models, such as Computer Numerically Controlled (CNC) manufacturing techniques and bed casting techniques. In particular, the present paper details the protocols developed to mill out a correct representation of the complex rock-fractured geometry of a closed channel which was obtained from Terrestrial Laser Scanners. The novelty of this scaled model production is the implementation of optical accesses in a closed (pressurized) hydraulic model, to allow for Particle Image Velocimetry measurements with a minimum impact on the reproduced roughness elements. The effectiveness of this production protocol is discussed in the context of modelling the roughness effects on the flow regime.
\end{abstract}

\section{Introduction}

Experimental research is an important field in environmental hydraulics and fluid mechanics, and the associated experimental methodologies have benefited from technological advances in the past decades. Data at high spatial and temporal resolution with a high accuracy can be acquired, allowing for, e.g., the validation of high-resolution numerical models, the derivation of novel theories and approaches, or for solving complex practical problems [1]. Thus, experimental research is and will remain a vital research tool that, together with numerical investigations, will help to further deepen our understanding of flow processes over complex boundaries [2].

Particularly scale models have proven to be an effective and cost-efficient tool to solve complex practical problems and to aid the design of hydraulic structures. Scale models have traditionally been built using a range of construction materials such as metals, plastics, wood,

* Corresponding author: pierre-yves.henry@ntnu.no 
sands, and cement. They rely on the principle of similitude [3] and the prototype topography needs to be reproduced at smaller spatial scales. For surface models, this has typically been achieved by forming a skeleton using cross-sectional profiles spaced at an arbitrary distance. As only the model elevations at the profiles can be guaranteed to match the scaled elevations of the prototype surface, details of the "real surface" in between the profiles as well as the main roughness characteristics may not be correctly reproduced. The inability to reproduce the prototype surface at each model point may influence the outcomes of the modelling study especially for surface models; short models of hydraulic structures are less affected. In order to achieve similitude of the bulk hydraulic characteristics between the model and the prototype, a model calibration is thus required for surface models to adjust the model surface roughness appropriately, for example by smoothing or roughening the surface with additional material such as sand or flexible strips [4, 7]. Moreover, differences in the roughness pattern may result in differences in the near bed turbulent flow patterns and boundary layer characteristics which, if significant, will contribute to both scale and laboratory effects [e.g., $1,5,6]$.

The purpose of the present paper is the description of novel methods for the reconstruction of complex topographies at smaller scales using novel model production techniques such as Computer Numerically Controlled (CNC) manufacturing methods and bed casting techniques. Both methodologies will be shortly reviewed in Section 2. Section 3 presents an example of the workflow required for the construction of a complex scale model. For this purpose, the reproduction of a complex rock-fractured geometry of a hydropower-tunnel is described for which the geometry was available from Terrestrial Laser Scanning [7]. The novelty of this scale model is the implementation of optical accesses in a closed (pressurized) hydraulic model to allow for Particle Image Velocimetry measurements with a minimized impact of the reproduced roughness elements.

\section{Recent advances in hydraulic model production}

Recent advances in the construction of hydraulic scale models are mostly represented by the application of casting methods or the application of CNC-based production techniques. Both will be briefly reviewed in the following.

\subsection{Casting techniques}

Casting is a process in which a mold is filled by a liquid material which is then allowed to solidify. The mold represents the counterpart of the cast, i.e. it is the negative of a body or a surface, and once the liquid material has solidified it is removed from the mold representing the cast. The mold can be constructed using different materials and methods (e.g. [8]) and the liquid material used for casting is typically synthetic resin. Due to the principle of the casting technique, it has mainly been used to directly reproduce surfaces at the 1:1 scale in hydraulic studies although molds can also be created at different spatial scales using 3Dprinting or milling (see below).

The casting technique has been employed recently to investigate the significance of interfacial hydraulics on the flow field over water worked gravel beds or the near bed turbulent flow field over complex (partly artificial) rough beds [e.g., 9-15]. The studies focusing on the significance of the interfacial hydraulics on the flow field made use of the fact that the surface layer of water worked beds can be reproduced with a high level of accuracy and that, at the same time, the porosity of the cast equals zero below the roughness troughs (Fig. 1). Thus, the comparison of the flow fields over the porous and casted bed, respectively, allows for an assessment of the role of interfacial hydraulics. The studies employing the casting technique also showed that care must be taken due to shrinkage of the 
cast during the hardening of the synthetic resin. In addition, the lowest layers of the cast (i.e. the transition from the interfacial sublayer to the subsurface layer) may not be as well reproduced as the top of the surface layer. The latter fact can be attributed to the molding process of water-worked beds with a porous subsurface as it cannot be ensured that the liquid used for the molding fills all the pore-spaces or that the fluvial material can be removed from the mold without distorting it before the creation of the cast $[9,10]$.

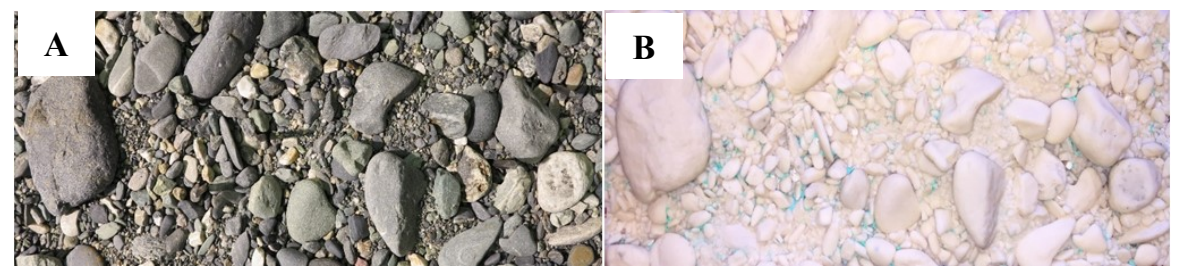

Fig. 1. View of a gravel bed armour layer (A) and its artificially reproduced counterpart without porous subsurface (resin cast, B) [13].

\subsection{D milling/printing}

CNC-manufacturing methods such as 3D-printing and 3D-milling require, in contrast to casting methods, Digital Elevation Models (DEMs) as an input. DEMs can nowadays be relatively easily obtained using remote sensing techniques such as laser scanning, sonar surveys or photogrammetric methods $[1,16]$. Given the availability of DEMs, both milling or printing can hence be used to reproduce fluvial or hydraulic structures at different spatial scales. Note that these methods allow also for the construction of molds by printing or milling the negative of the surface from which the surface can then be subsequently casted.

Producing an object by 3D-milling means that it is progressively shaped from a solid block of material using a CNC-controlled robot arm to which differently sized milling heads are attached. Different materials can be used for milling bodies or surfaces ranging from foams to metal alloys, and the accuracy of the milled surface or object depends on the accuracy of the DEM as well as the size of the milling head. 3D-milling of scale models is a relatively new technique and it has already been successfully applied to produce surfaces for air models [17], large scale hydraulic models of river reaches [pers. comm. T. Hüsener], models of bedrock channels [e.g., 18, 19], self-affine rough beds [20], or different hydraulic structures (e.g., at the hydraulic laboratory of the Norwegian University of Science and Technology).

$3 \mathrm{D}$ printing of scale models differs from milling as the process starts from a flat horizontal surface to which successive layers of a liquid material are applied by a small feeding conduct placed on a CNC-robot arm. This material must harden relatively fast so that the next layer can be applied. Different materials such as thermoplastics and resins are typically used for 3D-printing and have been used in different disciplines such as biomimetic [21], marine hydrodynamics [22], or microfluidics [23]. Concrete can also be used as printing material [24] and hence the 3D-printing technology, which has already successfully been applied in a couple of hydraulic investigations [e.g., 18, 25] may have a significant impact on the future production of hydraulic physical models.

Both milling and printing are limited to the spatial scale over which the CNC-machine can operate. However, models of a larger spatial extent can be constructed by milling or printing individual tiles which can then be combined to form the large-scale surface (the same applies for constructing models using the casting technique). In all cases, care needs to be taken in regard to buoyancy effects if the density of the material used for model production is lower than that of water. 


\section{Scaled model of the rock-fractured geometry of a closed channel}

This section describes the construction of a scale model of an unlined rock-blasted tunnel using CNC-milling. In general, rock-fractured surfaces are a special feature in hydraulics and are of particular importance in bedrock rivers and hydropower applications. Especially the adequate determination of friction losses associated with the wall roughness of unlined hydropower tunnels is challenging due to the complex surface roughness of blasted rocks and the lack of data on hydraulic resistance. It is against this background that a project was launched at the Norwegian University of Science and Technology to develop advanced predictive capabilities for the quantification of wall resistance in unlined hydropower tunnels, by combining scale model studies and numerical investigations [7]. In order to reproduce the prototype geometry as accurate as possible, it was decided to scan existing hydropower tunnels with a high spatial resolution and to use the scans for the milling of a scale model.

In the following, we describe the production of a 1:15 scale model of a short section of rock-blasted tunnel. The model will be used in subsequent studies for head loss and velocity measurements, to relate surface roughness characteristics to hydraulic resistance, to investigate the near bed flow pattern within the tunnel, and to provide data for the validation of numerical models.

\subsection{Generation of the DEM}

The DEM used for the construction of the model is based on the survey of a $120 \mathrm{~m}$ long and straight section of a hydropower tunnel belonging to the Lijtfossen hydropower plant in Norway. The scanning was carried out with a Leica C10 Terrestrial Laser Scanner, and the 230m long section was covered by scanning from 19 different positions (Nidaros Oppmåling AS). A local coordinate system was established by referencing the position of seven targets along the tunnel section with a total station (Leica TS15). The point clouds obtained at each position were post-processed and registered with the Iterative Closest Point algorithm implemented within the Leica Cyclone software package (version 9.1.2). The final scan of the tunnel section consisted of 42.927 .547 points, leading to an average resolution of around one point per $\mathrm{cm}^{2}$. Fig. $2 \mathrm{~A}$ shows an example of a scan based on the raw data, where the round circles on the tunnel invert reflect the position of the laser scanner.

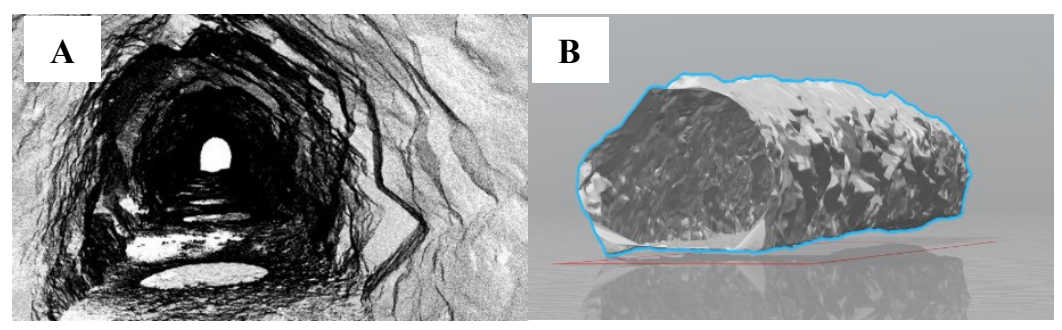

Fig. 2. View of the raw data from the scanned tunnel section (viewed with CloudCompare) and Digital Elevation Model created for the section of interest (viewed with SolidWorks).

The scan data were used to generate a DEM which was required for both the generation of meshes for CFD simulations and for milling the scaled model with Computer Aided Manufacturing (CAM). For this purpose, the 3D-Reshaper software package from Leica Geosystems was used. Initially, a model based on a patchwork of NURBS surfaces (Nonuniform rational B-spline, see e.g. [26]) was generated based on the reverse engineering approaches implemented in 3D-Reshaper, as spline surfaces are easier to handle with a CAM software [pers. comm. SINTEF Ocean]. However, due to the complexity of the rock surface, 
a grid with rather large tile size ( $1 \mathrm{~m}$ by $1 \mathrm{~m})$ had to be used to minimise the discontinuities between the patches. The large tile sizes lead to the undesired smoothing of the sharp rock edges which represent an essential part of the roughness. This problem was resolved by applying an alternative approach in which a fine triangular mesh was generated (1 million triangles, Fig. 2B). A comparison between the DEM obtained in this way and the original point cloud showed that $99.5 \%$ of the scanned points were less than $2 \mathrm{~cm}$ away from the mesh (at prototype scale, see [7]).

\subsection{Model production}

The tunnel was milled out of low-density polyvinylchloride (PVC) foam core material (Divinycell H60) in a large CNC milling facility at SINTEF Ocean in Trondheim, Norway (Fig.3). The base material consisted of $2440 \mathrm{~mm}$ long, $1220 \mathrm{~mm}$ wide and $85 \mathrm{~mm}$ thick foam plates which were glued together to form blocks of the required height for the milling. Using a CAM software, the DEM was divided into longitudinal sections defined by the plate length, and each section was divided into three segments to ensure that all the edges and cavities could be milled out properly (Fig. 3).

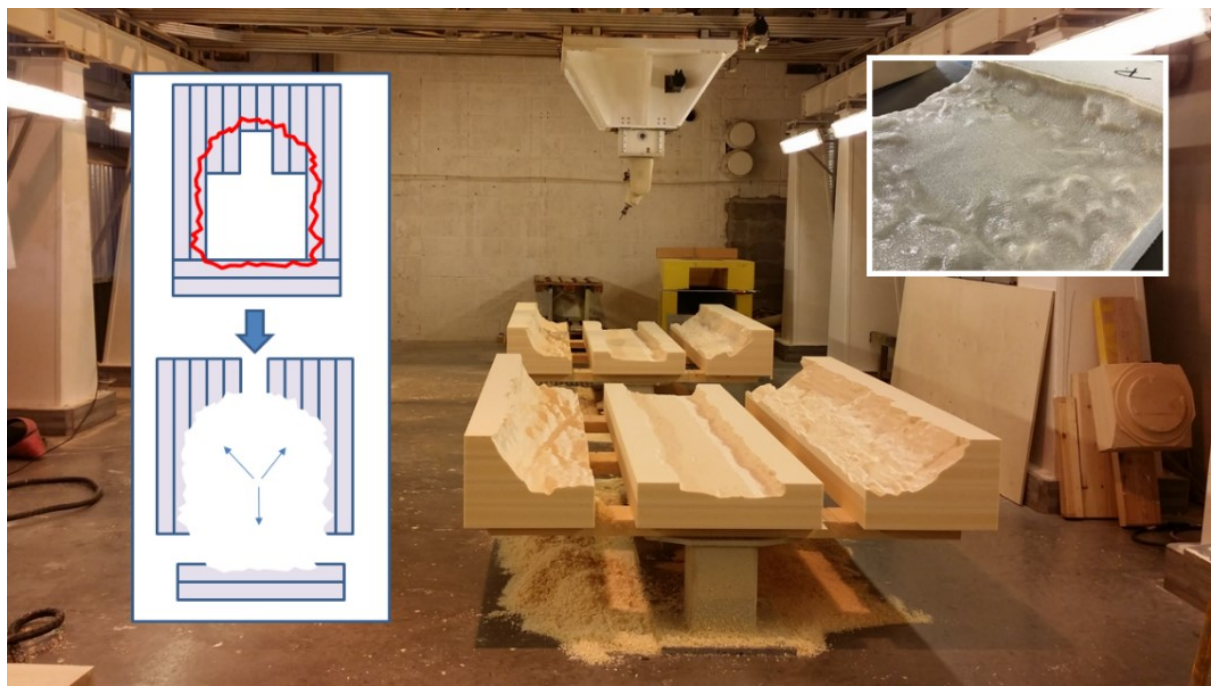

Fig. 3. Planning of the milling (SINTEF Ocean) and milling of the three 'sides' of two sections. Upright: milled and coated bottom, part of a tunnel sample.

Each tunnel segment was milled separately and the finest milling tool used was a $10 \mathrm{~mm}$ diameter ball-nosed endmill (diameters of $25 \mathrm{~mm}$ or $20 \mathrm{~mm}$ for rough milling). The effective milling time was appx 70-80h plus rigging and handling. After the milling was completed, the milled surfaces were sprayed with a polyester coating to reinforce the surface and improve water tightness before gluing the segments together to reconstruct the 3D roughness (see Fig. $3)$.

\subsection{Preparation for optical access}

The model construction included the possibility for optical access into the milled tunnel allowing for velocity measurements with Particle Laser Velocimetry (PIV). For this purpose, two window sets were manufactured, consisting of a $150 \mathrm{~mm} \times 250 \mathrm{~mm}$ window in the invert and an inclined window of the same size at the tunnel side. The openings for the windows were placed and designed using the scaled tunnel DEM (Fig. 4A and 4B). 


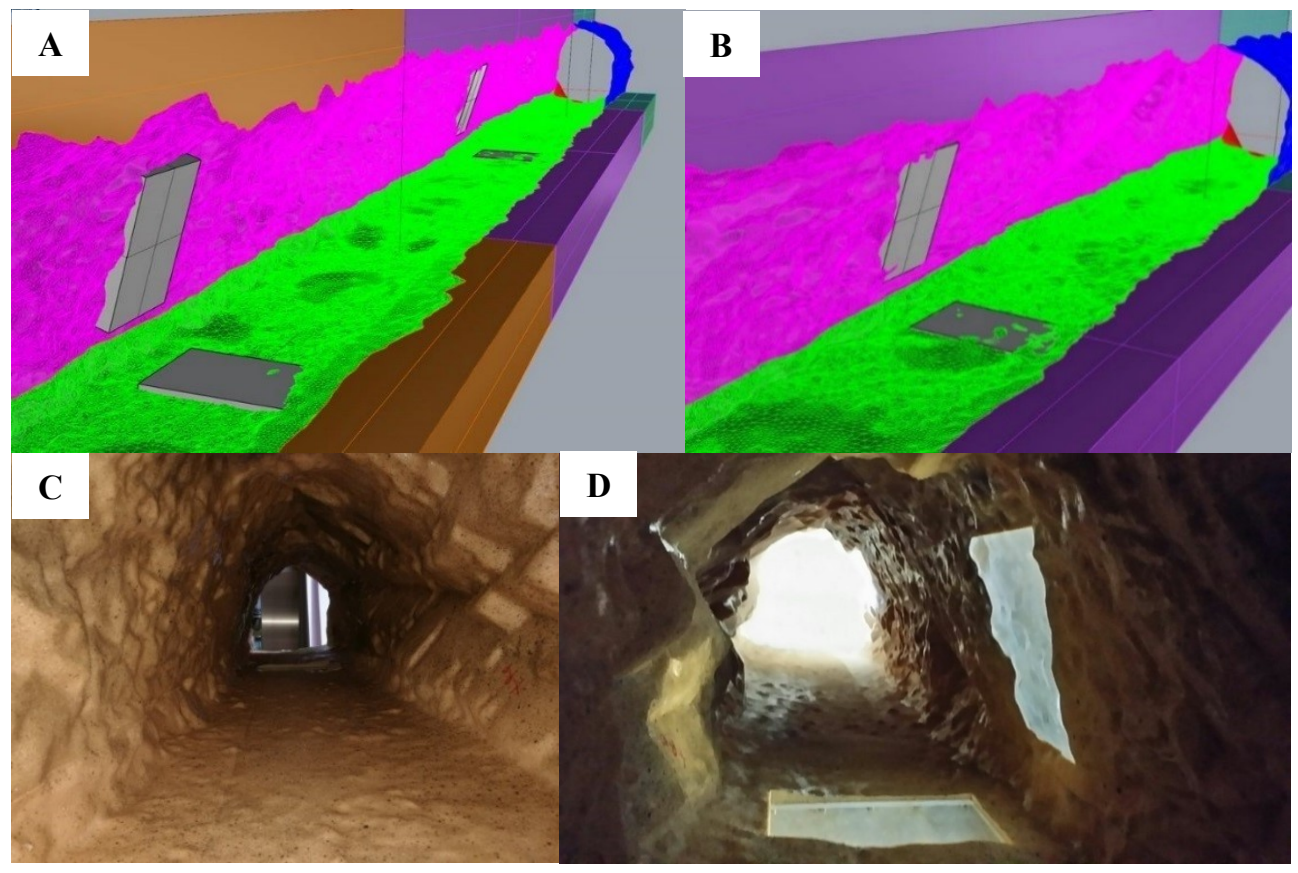

Fig. 4. Visualisation of the window position seen in the flow direction (realised with Rhino3D, by SINTEF Ocean) and view of the milled Litjfossen tunnel with the final milled side window.

The tunnel invert was rather smooth and was thus replaced by a smooth perspex window allowing for optical access with high-speed cameras. The tunnel walls, however, are rather rough and to avoid interference with the roughness pattern the side window was milled to match the wall roughness pattern (Fig. 4D). Horizontal thin grooves are milled out of the side windows to enable the formation of the laser sheet in the tunnel without any impact of the irregular window roughness. The design of the windows and their supporting structure was inspired from windows implemented in cavitation tunnels, to handle pressurise conditions.

\subsection{Model accuracy}

As mentioned before, the deviations between the original point cloud and the DEM were almost negligible. It can therefore be expected that most of the deviations between the milled tunnel and the downscaled prototype result from the milling process. The size of the finest milling head is the main parameter controlling the accuracy and the amount of "smoothing" involved in the milling process, i.e. it defines the loss of accuracy. On the other hand, the use of finer milling heads increases the production time (and cost) considerably so that the finest milling head is usually restricted to a diameter of $10 \mathrm{~mm}$ for large model sizes [pers. comm. SINTEF Ocean]. Working with a scale of $1: 15$, this implies difficulties in reproducing roughness elements in the order of $15 \mathrm{~cm}$ and less (in prototype scale). In order to quantify such deviations, the tunnel sides were scanned using the same total station and TLS as for the scan of the prototype. The scans were carried out prior to surface treatment and assembly of the tunnel sections. The variability in the reflective properties of the foam material in the infrared spectrum led to a bias in the scans of the right-hand side of the tunnel so that these scans could not be further analysed. The reference points on the left-hand side, on the other hand, could be accurately surveyed and no distortion in the scanned data could be detected. The point clouds obtained with the TLS had a density of about 70 points per $\mathrm{cm}^{2}$ (model scale), and could be registered with an accuracy close to the millimetre. 


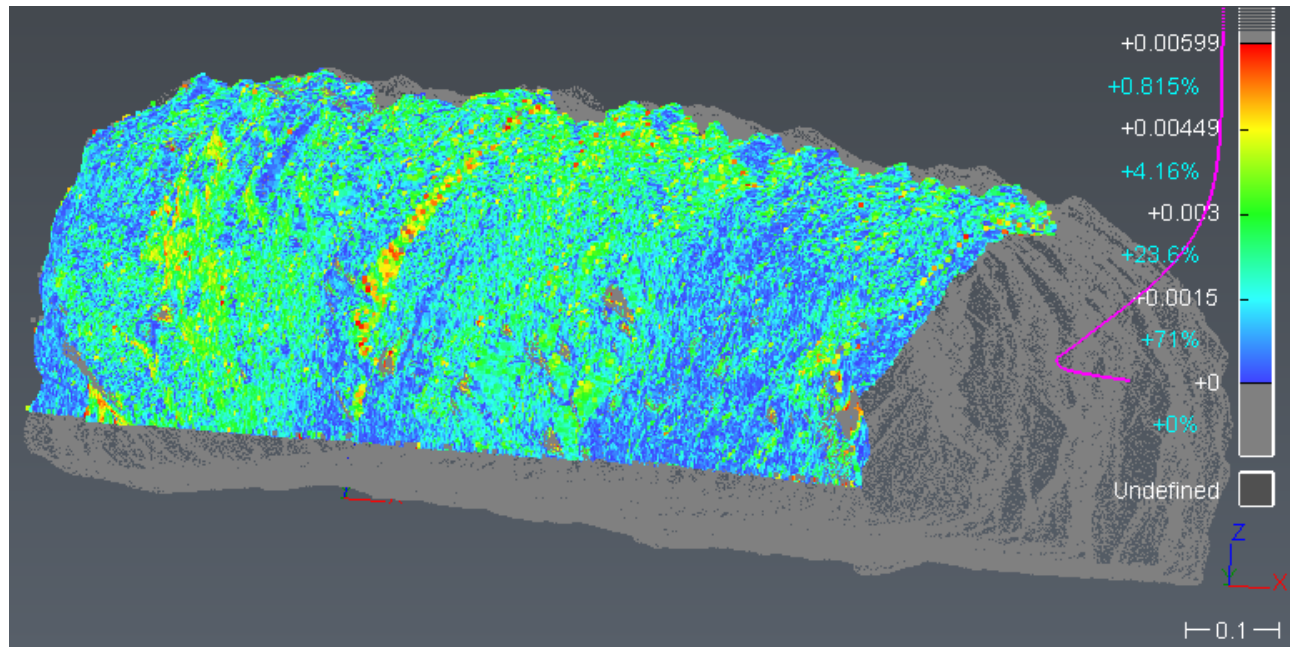

Fig. 5. Estimation accuracy from DEM to milling at the model scale. Scale in meters.

Fig. 5 illustrates the comparison between the scanned side of the model and the DEM used for production. It shows an acceptable accuracy with $94.6 \%$ of the scanned points being less than $3 \mathrm{~mm}$ away from the scaled DEM (more precisely, $71 \%$ of these points are in the range $\pm 1.5 \mathrm{~mm}$ from the scaled DEM). Larger deviations are typically observed in the troughs of larger roughness elements reflecting the limitations of the used milling head to reproduce such small cavities accurately. Nonetheless, the obtained accuracy of the surface features is remarkable and it may be assumed that the impact of these deviations may not be significant for the future hydraulic tests.

\section{Concluding remarks}

This paper provides an example of the use of novel CNC-based production techniques in experimental hydraulics, and details some important information on the accuracy of the technique at the different stages of the reproduction process. Taking the example of a scaled tunnel model, it was shown that the fine resolution of the technique ensures a high accuracy in the reproduction of the complex rock fractured geometry of the prototype. These CNCbased production methods offers thus, as highlighted in the first part of the paper, a solid base for further detailed geometrical and hydraulic roughness experimental investigations on any type of hydraulic structure, river reaches and other types of hydraulic components.

To this date, the milling technique has been mainly used to construct "short models" [e.g., 18], where a particular section or surface had a clear control of the flow processes, small scale air-models [e.g., 17] or artificial rough surfaces defined by specific scaling laws [e.g., $19,20]$. Tunnel Roughness is a project where the technique is used for the reconstruction of a complex $3 \mathrm{D}$ rough geometry in a so-called "long model", where the bed surface roughness plays a crucial role. The findings of this project will be used to investigate the geometrical downscaling of rough surfaces and the associated scaling of the hydraulic roughness (both surface and form roughness), i.e. the direct link of surface topography to flow resistance. This aspect remains unclear until today [27] and can be investigated with this technique, which will provide important information for future models in various hydraulic applications.

Finally, using high-resolution velocity and pressure measurements, such model tests will provide benchmark data sets for the validation of numerical investigations, as the identical topography can be used in the physical and numerical models. 
The Tunnel Roughness project is funded under the EnergiX-program of the Research Council of Norway (project no. 255318/E20) in collaboration with the Norwegian Water Resources and Energy Directorate, the Norwegian Hydropower Centre, and the industrial partners BKK and TrønderEnergi. This project is also affiliated with the research centre for environment-friendly energy research HydroCen.

\section{References}

1. M. Muste, J. Aberle, D. Admiraal, R. Ettema, M. Garcia, D. Lyn, V. Nikora, C. Rennie, Experimental Hydraulics: Methods, Instrumentation, Data Processing and Management (CRC Press, London, 2017)

2. P. Rowinski, Experimental Methods in Hydraulic Research, (Springer-Verlag, Berlin Heidelberg, 2011)

3. H. Rouse, S. Ince, History of Hydraulics (Iowa Inst. of Hydraulic Research, State university of Iowa, 1957)

4. V. Heller, J. Hydr. Res. 49, 3 (2011)

5. S. A. Hughes, Physical Models and Laboratory Techniques in Coastal Engineering (World Scientific, Singapore, 1993)

6. C. B. Webb, S. L. Barfuss, M. C. Johnson. J. Hydr. Res. 48, 260-264 (2010)

7. J. Aberle, P.Y. Henry, K. Bråtveit, in: Proc. ITA-WTC 2017 (NFF, Bergen, 2017)

8. T. Whelan, J. Goff, in: Molding of Thermosetting Plastics. (Springer, Boston, MA, 1990)

9. T. Buffin-Bélanger, I. Reid, S. Rice, J. H. Chandler, J. Lancaster, Earth Surf. Proc. Land. 28, 787-796 (2003)

10. S. Spiller, N. Rüther, in: Proc. $2^{\text {nd }}$ IAHR Europe, (TU München, Munich, 2012)

11. S. P. Rice, T. Buffin-Bélanger, I. Reid, Earth Surf. Proc. Land. 39, 184-199 (2014)

12. S. Spiller, N. Rüther, H. Friedrich. Water 7, 9 (2015)

13. C.U. Navaratnam, J. Aberle, S.M. Spiller, in: Proc. River Flow 2016, Taylor \& Francis Group, London, 2016)

14. C.U. Navaratnam, J. Aberle. Vann 52, 2 (2017)

15. J. R. Cooper, A. Ockleford, S. P. Rice, D. M. Powell, Earth Surf. Proc. Land. (In press)

16. S. Bertin, H. Friedrich, P. Delmas, E. Chan, G. Gimel'farb, Photo. Rec. 29, 146 (2014)

17. P. Faulhaber, Mitteilungsblatt der Bundesanstalt für Wasserbau, 90 (2007)

18. Ø. Pedersen, N. Rüther, Proc. Eng. Vol., 154, 433-440 (2016)

19. L. Andersson, I. Larsson, J. Hellstrom, P. Andreasson, A. Andersson, in: Proc. 6th IAHR Int. Symp. Hyd. Struct., (DigitalCommons@USU, Portland, 2016)

20. M. T. Stewart, S. M. Cameron, V. I. Nikora, A. Zampiron, I. Marusic. (In press)

21. L. Wen, J.C. Weaver, P.J.M. Thornycroft, G. V. Lauder. Bioi. \& Biom., 10, 6 (2015)

22. O. Turan, Y.K. Demirel, S. Day, T. Tezdogan, Transp. Res. Proc. 14, 1649-1658 (2016)

23. J. O’Connor, J. Punch, N. Jeffers, J. Stafford, Microfluid Nanofluid 19, 385 (2015)

24. Y.W.D. Tay, B. Panda, S.C. Paul, N.A. Noor Mohamed, M.J. Tan, K.F. Leong. Virt. Phys. Prot. 123 (2017)

25. R. A. Hodge, T. B. Hoey, J. Geophys. Res. Earth Surf., 121, 1578-1596 (2016)

26. W. Ma, J. P. Kruth, Int J Adv Manuf Technol 14, 918 (1998)

27. K. A., Flack, J. Fluid Mech., 842, 1-4 (2018) 